\title{
Quantifying the role of chaperones in protein translocation by computational modeling
}

\author{
Salvatore Assenza *, Paolo De Los Rios * and Alessandro Barducci * \\ Laboratoire de Biophysique Statistique, Ecole Polytechnique Fédérale de Lausanne, Lausanne, Switzerland
}

\section{OPEN ACCESS}

Edited by:

Annalisa Pastore,

King's College London, UK

Reviewed by:

Esko Oksanen,

European Spallation Source, Sweden

Piero Andrea Temussi,

Università di Napoli Federico II, Italy

${ }^{*}$ Correspondence:

Salvatore Assenza, Paolo De Los Rios and Alessandro Barducci,

Laboratoire de Biophysique

Statistique, Ecole Polytechnique

Fédérale de Lausanne, Cubotron 723,

CH-1015 Lausanne, Switzerland

salvatore.assenza@epfl.ch;

paolo.delosrios@epfl.ch

alessandro.barducci@epfl.ch

Specialty section:

This article was submitted to

Structural Biology, a section of the journal Frontiers in Molecular

Biosciences

Received: 06 January 2015

Paper pending published:

10 February 2015

Accepted: 28 February 2015

Published: 23 March 2015

Citation:

Assenza S, De Los Rios P and Barducci A (2015) Quantifying the role of chaperones in protein translocation

by computational modeling.

Front. Mol. Biosci. 2:8.

doi: 10.3389/fmolb.2015.00008
The molecular chaperone Hsp70 plays a central role in the import of cytoplasmic proteins into organelles, driving their translocation by binding them from the organellar interior. Starting from the experimentally-determined structure of the E. coli Hsp70, we computed, by means of molecular simulations, the effective free-energy profile for substrate translocation upon chaperone binding. We then used the resulting free energy to quantitatively characterize the kinetics of the import process, whose comparison with unassisted translocation highlights the essential role played by Hsp70 in importing cytoplasmic proteins.

Keywords: translocation, Hsp70, chaperones, molecular dynamics, free energy

\section{Introduction}

Molecular chaperones are protein machines that assist other proteins in various cellular processes. 70-kDa Heat Shock Proteins (Hsp70s) are possibly the most versatile chaperones, supervising a wide variety of cellular tasks (Mayer and Bukau, 2005) that range from disaggregation of stable protein aggregates (Diamant et al., 2000) to driving post-translational import of cytoplasmic proteins into organelles (Matlack et al., 1999; Neupert and Brunner, 2002; Liu et al., 2014). Notably, Hsp70s play a fundamental role in the import of proteins into mitochondria because the majority of mitochondrial proteins are actually encoded in nuclear DNA, synthesized in the cytosol and only post-translationally imported into the organelles. Protein import takes place through a proteinaceous pore that spans the two mitochondrial membranes by way of the outer (TOM) and inner (TIM) membrane pore complexes (Neupert and Brunner, 2002). According to the current view, an ATP-consuming import motor located into the mitochondrial matrix drives the inward translocation of nuclear-encoded proteins. Mitochondrial Hsp70 ( $\mathrm{mtHsp} 70)$ is the central element of this motor: it is recruited by the TIM complex on the matrix side through interactions with the TIM44 protein, which is part of the pore, and with the pore-associated PAM16/18 proteins. The latter contain a J domain, whose role is to dramatically enhance the ATP-hydrolysis rate of Hsp70, thus increasing by orders of magnitude its affinity for substrates. The ATP-driven binding of the chaperones to incoming proteins ultimately drives their translocation.

The structure of Hsp70 is highly conserved (Zuiderweg et al., 2013) and consists of two large domains connected by a small flexible linker (see Figure 1). Specifically, the Nucleotide Binding Domain (NBD) is the ATPase unit of the chaperone, while the Substrate Binding Domain (SBD) directly interacts with specific sites on the incoming protein. These binding sites are frequently found in protein sequences, so that multiple chaperones are likely to bind the same substrate.

The precise mechanism by which Hsp70 exerts its pulling action has been debated in the literature and several models have been proposed (Glick, 1995; Neupert and Brunner, 2002; De Los Rios et al., 2006). The Brownian ratchet (Neupert and Brunner, 2002) assumes that, thanks to the 


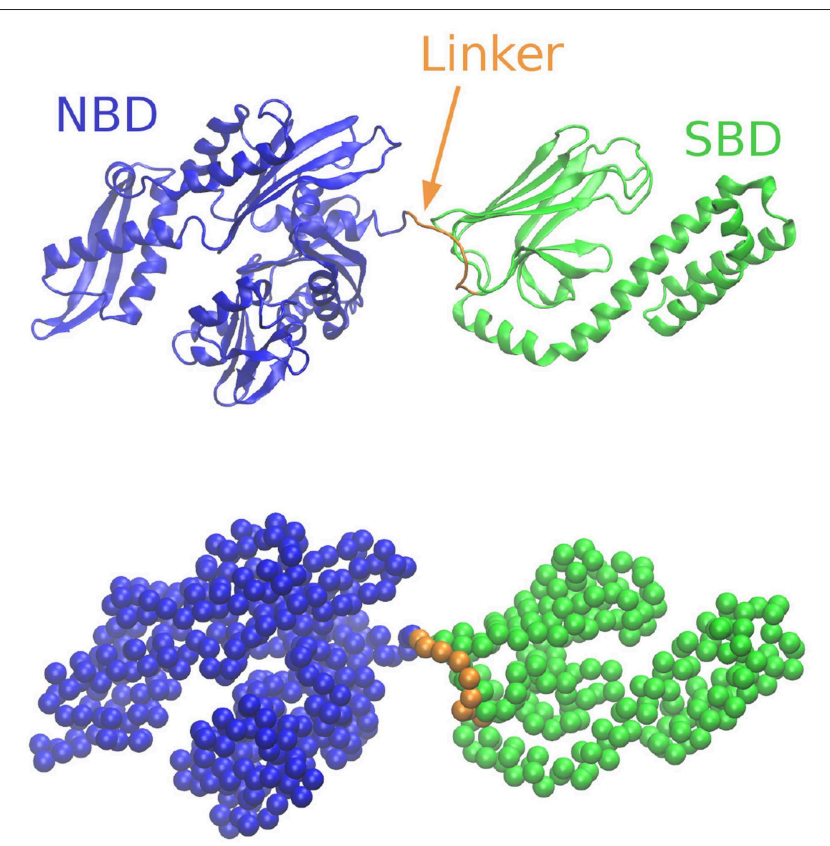

FIGURE 1 | Cartoon representation of the Hsp70 chaperone at the secondary-structure level (top) and corresponding coarse-grained model considered in this work (bottom).

chaperone large size, Hsp70 binding prevents the retrotranslocation of the substrate into the pore, thus biasing the random fluctuations toward the matrix. Alternatively, according to the power stroke (Glick, 1995) the chaperone actively pulls the incoming protein by using TIM as a fulcrum. Later, according to the entropic pulling model (De Los Rios et al., 2006), it was shown that an active force naturally emerges from a realistic physical description of the Brownian ratchet, thus reconciling the two views (Goloubinoff and De Los Rios, 2007). Indeed, the excluded volume of the chaperone, besides preventing retrotranslocation, reduces the conformational space available to the incoming protein, thus decreasing its entropy. This reduction depends on the length of the imported fragment of the substrate, therefore resulting in a free-energy gradient which favors the import.

In the present work, we evaluate this thermodynamic force in an effective one-dimensional space where the state of the system is represented by the number $n$ of imported residues. In order to do so, for each value of $n$ we compute the effect of chaperone binding on the free energy of the system by means of coarsegrained Molecular Dynamics (MD) simulations. This result is then used to devise a simplified yet quantitative analysis of the import, described as a one-dimensional diffusion process on the computed free-energy landscape.

\section{Materials and Methods}

\subsection{Details of MD Simulations}

We coarse-grained both the substrate and the chaperone by considering one interaction site per residue centered on the $\mathrm{C}_{\alpha}$ atom. Residue-residue excluded-volume interactions were modeled with a repulsive Lennard-Jones potential with parameters $\sigma=3.8 \AA$ and $\varepsilon=3 k_{B} T$. The substrate was modeled by using the local flexible potential introduced in Ghavami et al. (2013). Within this force field, the elastic properties of a coarse-grained unfolded protein are described by means of harmonic $\mathrm{C}_{\alpha}-\mathrm{C}_{\alpha}$ bonds and sequence-specific bending and torsional potentials. Particularly, the sequence dependence is introduced by considering a simplified three-letters description, where the eventual presence of glycine or proline amino acids is explicitly accounted for, while all the other residues are considered equivalent. In the present work, for simplicity we focus on glycine- and proline-free substrates, thus making use of the functions denoted as $\mathrm{O}-\mathrm{X}-\mathrm{Y}$ and $\mathrm{X}-\mathrm{X}$ in Ghavami et al. (2013) for the bending and torsional contributions, respectively. The experimental structure of ADPbound Hsp70 (Bertelsen et al., 2009) (PDB: 2KHO) was used to model the chaperone. In particular, the NBD (residue 4-387) and SBD (residue 397-603) were treated as rigid bodies, while the flexibility of the interdomain linker was accounted for by means of the potential described above. In order to reproduce a correct chaperone-substrate arrangement, we took advantage of the substrate-bound X-ray structure of DnaK SBD (PDB: 1DKX Zhu et al., 1996). MD simulations were performed using LAMMPS code (Plimpton, 1995$)$ at constant temperature $(T=300 \mathrm{~K})$ by means of a Langevin thermostat with damping parameter equal to $100 \mathrm{fs}$ and using an integration timestep of $10 \mathrm{fs}$. For each value of $n$ in the range $8 \leq n \leq 26$ we performed a MD simulation of $5 \cdot 10^{10}$ timesteps (examples of the convergence of the ratio $\mathcal{Z}_{70}(n) / \mathcal{Z}(n)$ are reported in the Figure $\mathbf{S 1}$-Supplementary Material), and the error on the free-energy profile was estimated by block averaging (Frenkel and Smith, 2002).

\subsection{Details of the Stochastic Simulations}

The import process was simulated by means of a Monte Carlo (MC) algorithm driven by the free-energy landscape $F_{\text {import }}$, as determined from the sum of the chaperones pulling contribution computed by means of the MD simulations and the unfolding free energy $F_{u}$. The latter is modeled as a sigmoidal function

$$
F_{u}\left(n_{\mathrm{in}}\right)=\frac{F_{u}^{\max }}{1+\exp \left[5-10\left(n_{\mathrm{in}}-10\right) / \delta n\right]},
$$

where $n_{\text {in }}$ is the total number of imported residues, and $F_{u}^{\max }$ and $\delta n$ are tunable parameters representing the total free energy of unfolding and the cooperativity of the unfolding process, respectively (see Figure 4 top). For a system at position $n_{\text {in }}$, a trial move was attempted to either $n_{\text {in }}+1$ or $n_{\text {in }}-1$ with equal probability and accepted according to the Metropolis criterion based on the free energy $F_{\text {import }}$. To capture the sequence heterogeneity of the proteome, for each choice of $F_{u}^{\max }$ and $\delta n$ we generated 25 independent binding-site distributions, with the sole prescription that the average distance between consecutive binding sites was 35 residues as indicated by experiments (Rüdiger et al., 1997). For every distribution we performed 10 independent realizations of the import process. Average import times were estimated by counting the total number of MC timesteps needed 
for the translocation process to be completed. This protocol is justified by the fact that MC simulations correspond to overdamped Langevin dynamics when only local moves are considered (van Kampen, 1992; Tiana et al., 2007). Rescaling the obtained import times by the acceptance rate, as proposed in Sanz and Marenduzzo (2010), did not affect the results, because of the large fraction of accepted moves observed in all the simulations (> 95\%).

\section{Results}

Protein import into organelles has been previously modeled as a one-dimensional stochastic process in the space of the imported residues (Elston, 2000, 2002; Liebermeister et al., 2001). In the present context, this protocol is justified by the timescale separation among substrate conformational dynamics, chaperone binding/unbinding and overall import. Indeed, the typical reconfiguration time of an unfolded protein $(\sim 100 \mathrm{~ns}$ Soranno et al., 2012) is extremely fast compared to the experimentallydetermined timescale for protein import into mitochondria (order of minutes Lim et al., 2001). Effects arising from substrate conformational dynamics, such as the chaperone-induced entropy reduction, can be thus conveniently represented as effective free-energy profiles influencing the import dynamics. Moreover, the import timescale is also significantly slower than chaperone binding but faster than chaperone unbinding at physiological conditions. Indeed, according to the current understanding of the biochemical cycle of Hsp70 (Zuiderweg et al., 2013; De Los Rios and Barducci, 2014), ATP-bound chaperones associate with the substrate with a timescale equal to $\sim 10^{-2}$ $\mathrm{s}$ (as estimated from a Hsp70-peptide association rate equal to $4.5 \times 10^{5} \mathrm{M}^{-1} \mathrm{~s}^{-1}$ Schmid et al., 1994 and a chaperone concentration of $70 \mu \mathrm{M}$ in mitochondria Liu et al., 2003), while dissociation takes place from the ADP-bound state, over timescales $\sim 10^{3}$ s (Mayer et al., 2000). In the mitochondrial matrix chaperone-substrate dissociation is accelerated by the presence of nucleotide exchange factors, that catalyze the release of ADP and the ensuing rebinding of ATP. However, ATP hydrolysis is greatly enhanced in the proximity of the pore by poreassociated J-domain proteins, resulting into ultra-affinity for the substrate (De Los Rios and Barducci, 2014) and thus into an immediate replacement of the dissociated chaperone. This suggests that, to our purposes, we can assume that a chaperone immediately and irreversibly binds each exposed binding site as soon as it is imported. As a consequence, for the present purposes the number $n$ of substrate residues that have been imported into the mitochondrial matrix is a convenient coordinate to describe the system, whose dynamics can be modeled as a diffusion process on the corresponding free-energy landscape.

\subsection{Free Energy Calculation}

The effect of the size of the chaperone is two-fold. On the one hand, bound Hsp70 prevents the retrotranslocation of the substrate beyond its binding point (Brownian ratchet model Neupert and Brunner, 2002). On the other hand, the size of the chaperone leads also to a reduced number of sampled conformations (entropic pulling De Los Rios et al., 2006), an effect not accounted for by the Brownian ratchet as it was originally conceived, but nonetheless intimately related to the same physical mechanism. For example, in the absence of Hsp70 the two substrate conformations shown in the top panel of Figure $\mathbf{2}$ are both sterically allowed. However, upon chaperone binding the conformation on the right would result into an overlap between the membrane and Hsp70 (bottom panel in Figure 2), and it is therefore never sampled by the substrate when the chaperone is present. The free energy difference due to the loss of entropy is given by $\Delta F_{c}(n)=-k_{B} T \log \left(\mathcal{Z}_{70}(n) / \mathcal{Z}(n)\right)$, where $\mathcal{Z}_{70}(n)$ and $\mathcal{Z}(n)$ are the partition functions of the substrate with and without a bound chaperone, $k_{B}$ is the Boltzmann constant and $T$ the temperature (when enthalpic contributions are not taken into account, the partition functions reduce to the number of sampled conformations, thus falling back to the original formulation of the entropic-pulling free energy De Los Rios et al., 2006). Here, we computed the free energy difference $\Delta F_{c}(n)$ by estimating the ratio $\mathcal{Z}_{70}(n) / \mathcal{Z}(n)$ for $n$ in $8 \leq n \leq 26$ with multiple coarse-grained MD simulations. The substrate was modeled as a $n$-residues flexible chain with the position of the $n$th residue constrained on the inner mithocondrial membrane, represented here as a flat wall acting only on the substrate residues (see Materials and Methods for additional details). As a consequence, the system could sample configurations involving an overlap between the membrane and the chaperone (see bottom-right panel in Figure 2). With this strategy, we could estimate the ratio $\mathcal{Z}_{70}(n) / \mathcal{Z}(n)$ as the fraction of time spent by the system in physically-acceptable, i.e., non-overlapping, configurations. Particularly, we focused on $n \geq 8$ in order to allow the exposure of a complete binding site. From the computed values of $\mathcal{Z}_{70}(n) / \mathcal{Z}(n)$, we could retrieve the free energy $\Delta F_{c}(n)$ as a function of $n$, as reported in Figure 3. As expected, shorter imported fragments resulted into a larger fraction of rejected conformations, i.e., larger values of $\Delta F_{c}$, thus leading to a free-energy gradient favoring the import of the protein. The slope of the entropic-pulling free-energy profile corresponds to the thermodynamic pulling force exerted by a bound chaperone along $n$ (Figure 3 inset). This force is in the piconewton range, starting from around $15 \mathrm{pN}$ and decreasing as $n$ increases. Remarkably, these results agree qualitatively with previous estimates based on strongly simplified representations of the system (De Los Rios et al., 2006), thus suggesting that comparable thermodynamic forces could be obtained by the same entropic pulling mechanism for macromolecules of similar size.

\subsection{Stochastic Simulations of the Import Process}

We modeled the import of cytoplasmic proteins as a onedimensional stochastic process depending on the number $n_{\text {in }}$ of imported amino acids. The effective free-energy profile guiding the system evolution results from protein unfolding (Eilers and Schatz, 1986) and active chaperone pulling (Lim et al., 2001). Assuming a two-states folding behavior, a convenient choice to model the unfolding contribution to the free-energy landscape is a tunable sigmoidal function $F_{u}\left(n_{\text {in }}\right)$ (see Materials and Methods), depending on two parameters that measure the 


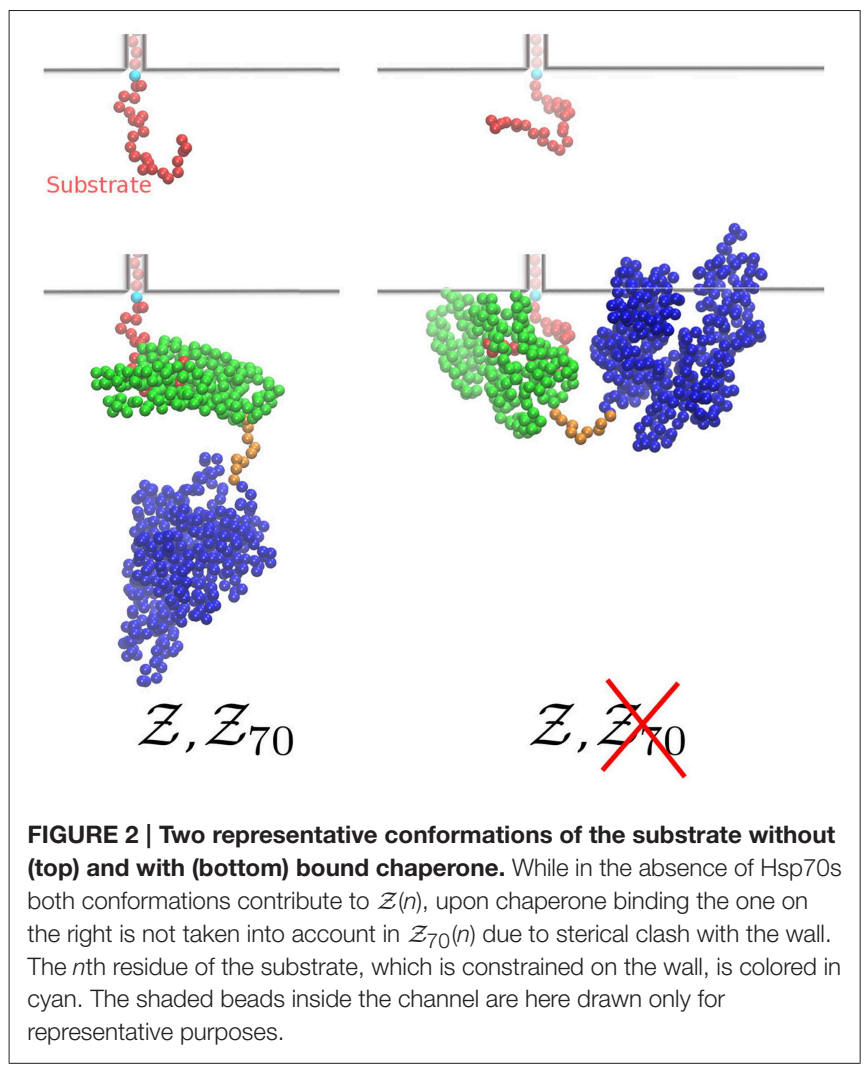

total unfolding free energy $\left(F_{u}^{\max }\right)$ and the cooperativity of the unfolding process $(\delta n)$, with smaller values of $\delta n$ corresponding to higher cooperativity (top panel in Figure 4). By tuning these parameters, the formula can account for the wide variety of imported proteins (Wilcox et al., 2005). The pulling action of the chaperone was modeled taking advantage of the freeenergy profile determined from molecular simulations. Particularly, we assumed here that: (i) Hsp70s associate with each binding site as soon as it emerges from the pore, since they are targeted at the TIM pore exit by specific interactions (Neupert and Brunner, 2002); (ii) we considered only the contribution arising from the Hsp70 closest to the pore, taking into account the relatively fast decrease of the slope of $\Delta F_{c}$ (see Figure 3) and the average frequency of binding sites (one every 35 amino acids Rüdiger et al., 1997). Therefore, we added to the unfolding free-energy $F_{u}\left(n_{\text {in }}\right)$ the chaperone contribution $\Delta F_{c}\left(n_{\text {in }}-\right.$ $n_{B}$ ), with $n_{B}$ corresponding to the position of the binding site closest to the pore, measured from the matrix terminus of the substrate.

As an example, in the bottom panel of Figure 4 we illustrate the evolution of the free-energy landscape during the import process of a protein with $F_{u}^{\max }=5 k_{B} T, \delta n=100$ and two binding sites at $n_{B}=0$ (i.e., at the matrix terminus) and $n_{B}=28$. At the beginning of the import process, no chaperone is bound to the substrate and the import free energy is simply given by $F_{\text {import }}\left(n_{\text {in }}\right)=F_{u}\left(n_{\text {in }}\right)$ (red dashed curve). As soon as the first binding site is imported, a chaperone molecule binds the substrate and its contribution $\Delta F_{c}$ is added to $F_{u}\left(n_{\text {in }}\right)$ starting from

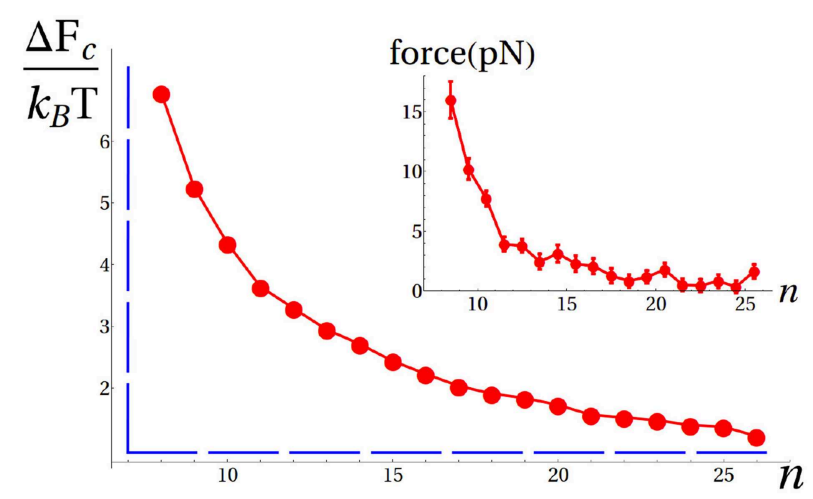

FIGURE 3 | Free-energy profile due to chaperone binding as a function of $\boldsymbol{n}$ (red circles). Error bars are smaller than the size of the symbols. The dashed blue line depicts the free-energy landscape predicted by the original Brownian ratchet, where the only effect of the chaperone is to prevent retrotranslocation beyond the binding site (infinite wall). In the inset we report the thermodynamic force corresponding to the computed free-energy landscape.

the binding site $n_{B}=0: F_{\text {import }}\left(n_{\text {in }}\right)=F_{u}\left(n_{\text {in }}\right)+\Delta F_{c}\left(n_{\text {in }}\right)$ (purple continuous curve). Finally, after the second binding site $\left(n_{B}=28\right)$ is imported, another chaperone binds and the resulting free energy is $F_{\text {import }}\left(n_{\text {in }}\right)=F_{u}\left(n_{\text {in }}\right)+\Delta F_{c}\left(n_{\text {in }}-28\right)$ (orange dot-dashed curve).

Following this approach, we computed the average import time (see Materials and Methods) of 300-residue proteins for different values of $\delta n$ and a range of $F_{u}^{\max }$ corresponding to the stability of a large fraction of the proteome (Ghosh and Dill, 2010). In absence of Hsp70 assistance, the system must invariably overcome a free-energy barrier, and the average import time $\tau_{0}$ increases exponentially with $F_{u}^{\max }$, independently of cooperativity (Figure 5 top). In all the considered cases, the average import time for the chaperone-assisted process, $\tau_{C}$, is sensibly smaller than $\tau_{0}$ (Figure 5 center). The chaperone pulling force reduces but does not completely eliminate the unfolding freeenergy difference for stable proteins (large $F_{u}^{\max }$ ), as in the case of the representative process shown in the bottom panel of Figure 4. In this regime, the import is still an activated process, and the average times increase exponentially with $F_{u}^{\max }$. Conversely, the pulling action of Hsp70 dominates over the unfolding contribution for marginally stable proteins ( $\operatorname{small} F_{u}^{\max }$ ), thus resulting in values of $\tau_{C}$ comparable to what found for the extreme case $F_{u}^{\max }=0$. The import kinetics is further modulated by $\delta n$, with high cooperativity (small $\delta n$ ) resulting in longer translocation times.

In the bottom panel in Figure 5 we illustrate the chaperoneinduced kinetic advantage by reporting the ratio $\tau_{0} / \tau_{C}$. This ratio ranges from a 10 -fold gain for marginally stable proteins to $10^{3}$ for extremely stable and non-cooperative substrates, with the majority of the proteome $\left(F_{u}^{\max } \geq 8 k_{B} T\right.$ Ghosh and Dill, 2010) accelerated at least 100 times. If we take into account that protein import into mithocondria has been measured to happen in the timescale of several minutes (Lim et al., 2001), our model indicates that the translocation process in 


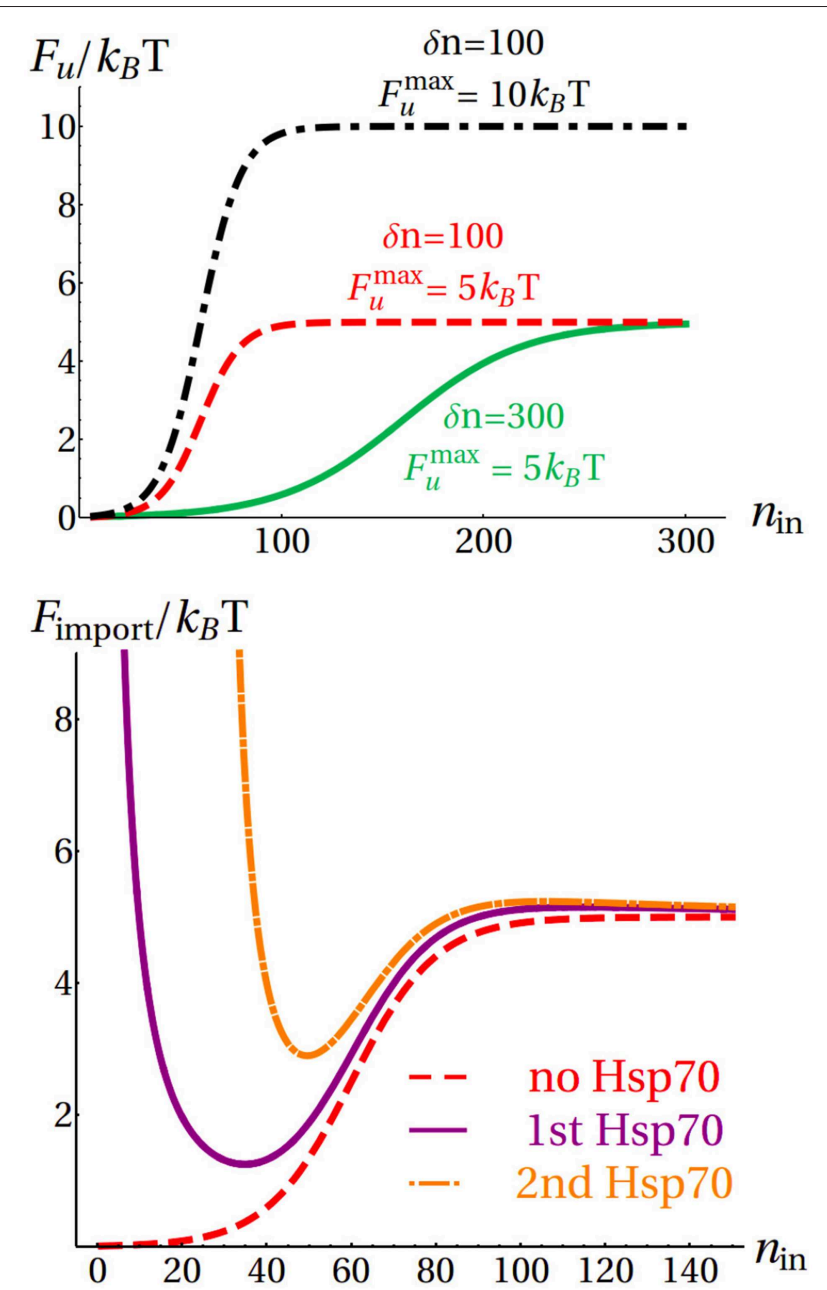

FIGURE 4 | Top: Influence of the parameters $F_{u}^{\max }$ and $\delta n$ on the unfolding free-energy. Bottom: Evolution of the total free-energy $F_{\text {import }}$ in a representative import process.

the absence of chaperones would probably extend to hours or days. Since such a slow process would clearly be incompatible with the average lifespan of proteins and the duration of the cell cycle, our results provide a molecular basis to support the essential role of chaperones in the in vivo import process.

\section{Conclusions}

To summarize, in this work we derived a free-energy profile for the import process based on a molecular description of Hsp70 that rationalizes the requirement for chaperone assistance in mitochondrial protein import observed in experiments. Naturally, a more precise quantitative estimation could be obtained by considering a more refined representation of the system, i.e., going beyond the coarse-grained model at residue level resolution employed in this work and considering also other interactions than the excluded volume. The
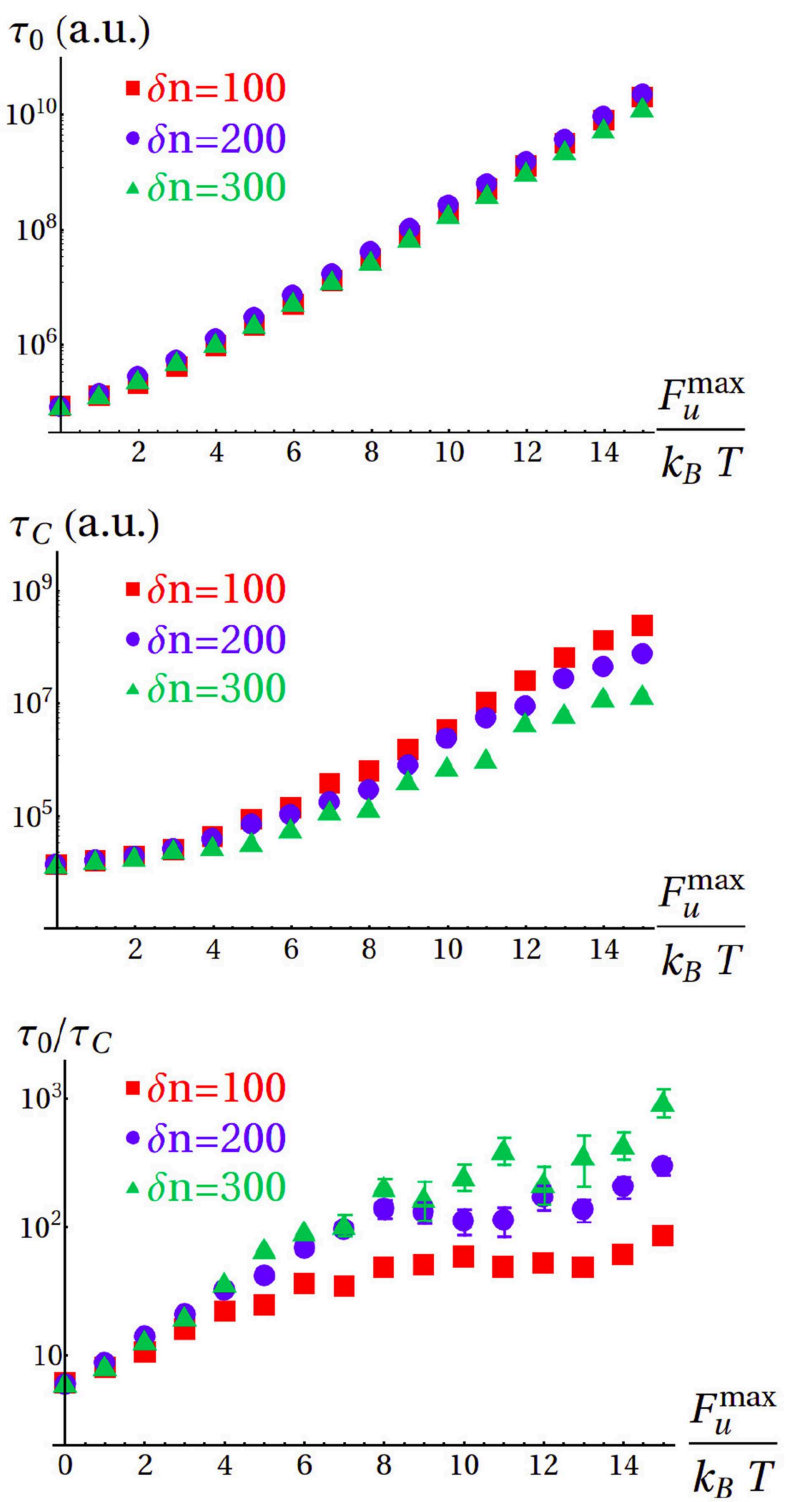

FIGURE 5 | Top: Average import times in the absence of chaperone $\left(\tau_{0}\right)$ as a function of $F_{U}^{\max }$ for different cooperativities (values for $F_{U}^{\max } \geq 12 k_{B} T$ were extrapolated by fitting the data in the range $4 k_{B} T \leq F_{u}^{\max } \leq 11 k_{B} T$ with exponential functions). Center: Average import times in the presence of Hsp70 $\left(\tau_{C}\right)$ for the same cases as in the top panel. Bottom: Acceleration of the process due to the assistance of $\mathrm{Hsp} 70$, expressed as the ratio $\tau_{0} / \tau_{C}$.

present results can be applied to other cases of Hsp70-driven translocation, namely protein import into ER (Matlack et al., 1999) and chloroplasts (Liu et al., 2014). In the ER case, in particular, the pore is much simpler than in mitochondria, as it spans just a single membrane. The presence of Sec63, a pore-associated protein containing a J domain, ensures also in this context that the extended ATP-driven Hsp70 ultraffinity prevails in the competition against other translocation counterproductive interactions (Scidmore et al., 1993). Moreover, this approach based on the combination of molecular 
simulations and kinetic modeling can be easily extended to other Hsp70-mediated cell processes. In particular, this freeenergy picture could help to understand some recent results pointing toward a fundamental role of Hsp70 in preventing the stalling of translation at ribosomes (Liu et al., 2013; Shalgi et al., 2013). Owing to the universality of the interaction responsible for the effects studied here, namely excluded volume, the same principles could apply to similar processes driven by other biomolecules.

\section{Author Contributions}

SA, PDLR, and AB designed and performed research, analyzed the results and wrote the paper.

\section{References}

Bertelsen, E. B., Chang, L., Gestwicki, J. E., and Zuiderweg, E. R. (2009). Solution conformation of wild-type E. coli Hsp70 (DnaK) chaperone complexed with ADP and substrate. Proc. Natl. Acad. Sci. U.S.A. 106, 8471-8476. doi: 10.1073/pnas.0903503106

De Los Rios, P., and Barducci, A. (2014). Hsp70 chaperones are non-equilibrium machines that achieve ultra-affinity by energy consumption. Elife 3:e02218. doi: 10.7554/eLife.02218

De Los Rios, P., Ben-Zvi, A., Slutsky, O., Azem, A., and Goloubinoff, P. (2006). Hsp70 chaperones accelerate protein translocation and the unfolding of stable protein aggregates by entropic pulling. Proc. Natl. Acad. Sci. U.S.A. 103, 6166-6171. doi: 10.1073/pnas.0510496103

Diamant, S., Ben-Zvi, A. P., Bukau, B., and Goloubinoff, P. (2000). Size-dependent disaggregation of stable protein aggregates by the DnaK chaperone machinery. J. Biol. Chem. 14, 21107-21113. doi: 10.1074/jbc.M001293200

Eilers, M., and Schatz, G. (1986). Binding of a specific ligand inhibits import of a purified precursor protein into mitochondria. Nature 322, 228-232. doi: $10.1038 / 322228 \mathrm{a} 0$

Elston, T. (2000). Models of post-translational protein translocation. Biophys. J. 79, 2235-2251. doi: 10.1016/S0006-3495(00)76471-X

Elston, T. (2002). The Brownian ratchet and power stroke models for posttranslational protein translocation into the endoplasmic reticulum. Biophys. J. 82, 1239-1253. doi: 10.1016/S0006-3495(02)75480-5

Frenkel, D., and Smith, B. (2002). Understanding Molecular Simulation, 2nd Edn. San Diego, CA: Academic Press.

Ghavami, A., der Giessen, E. V., and Onck, P. R. (2013). Coarse-grained potentials for local interactions in unfolded proteins. J. Chem. Theory Comput. 9, 432-440. doi: 10.1021/ct300684j

Ghosh, K., and Dill, K. (2010). Cellular proteomes have broad distributions of protein stability. Biophys. J. 99, 3996-4002. doi: 10.1016/j.bpj.2010. 10.036

Glick, B. S. (1995). Can Hsp70 proteins act as force-generating motors? Cell 80, 11-14. doi: 10.1016/0092-8674(95)90444-1

Goloubinoff, P., and De Los Rios, P. (2007). The mechanism of Hsp70 chaperones: (entropic) pulling the models together. Trends Biochem. Sci. 32, 372-380. doi: 10.1016/j.tibs.2007.06.008

Liebermeister, W., Rapoport, T. A., and Heinrich, R. (2001). Ratcheting in posttranslational protein translocation: a mathematical model. J. Mol. Biol. 305, 643-656. doi: 10.1006/jmbi.2000.4302

Lim, J. H., Martin, F., Guiard, B., Pfanner, N., and Voos, W. (2001). The mitochondrial Hsp70-dependent import system actively unfolds preproteins and shortens the lag phase of translocation. EMBO J. 20, 941-950. doi: 10.1093/emboj/20.5.941

Liu, B., Han, Y., and Qian, S. (2013). Cotranslational response to proteotoxic stress by elongation pausing of ribosomes. Mol. Cell 49, 453-463. doi: 10.1016/j.molcel.2012.12.001

\section{Funding}

The authors thank the Swiss National Science Foundation for support under the grant 200021-138073 (SA and PDLR) and the Ambizione fellowship program (AB).

\section{Supplementary Material}

The Supplementary Material for this article can be found online at: http://www.frontiersin.org/journal/10.3389/fmolb. 2015.00008/abstract

Supp. Figure 1 | Evolution of the ratio $\mathcal{Z}_{70}(n) / \mathcal{Z}(n)$ as a function of MD timesteps, for $\boldsymbol{n}=7$ (top panel), $\boldsymbol{n}=16$ (middle panel) and $\boldsymbol{n}=25$ (bottom panel).

Liu, L., McNeilage, R. T., Shi, L. X., and Theg, S. M. (2014). ATP requirement for chloroplast protein import is set by the Km for ATP hydrolysis of stromal Hsp70 in Physcomitrella patens. Plant Cell 113, 1246-1255. doi: $10.1105 /$ tpc.113.121822

Liu, Q., D’Silva, P., Walter, W., Marszalek, J., and Craig, E. A. (2003). Regulated cycling of mitochondrial Hsp70 at the protein import channel. Science 300, 139-141. doi: 10.1126/science.1083379

Matlack, K. E., Misselwitz, B., Plath, K., and Rapoport, T. A. (1999). BiP acts as a molecular ratchet during posttranslational transport of prepro-alpha factor across the ER membrane. Cell 97, 553-564. doi: 10.1016/S0092-8674(00) 80767-9

Mayer, M. P., and Bukau, B. (2005). Hsp70 chaperones: cellular functions and molecular mechanism. Cell. Mol. Life Sci. 62, 670-684. doi: 10.1007/s00018004-4464-6

Mayer, M. P., Schröder, H., Rüdiger, S., Paal, K., Laufen, T., and Bukau, B. (2000). Multistep mechanism of substrate binding determines chaperone activity of Hsp70. Nat. Struct. Biol. 7, 586-593. doi: 10.1038/ 76819

Neupert, W., and Brunner, M. (2002). The protein import motor of mitochondria. Nat. Rev. Mol. Cell. Biol. 3, 555-565. doi: 10.1038/nrm878

Plimpton, S. (1995). Fast parallel algorithms for short-range molecular dynamics. J. Comput. Phys. 117, 1-19. doi: 10.1006/jcph.1995.1039

Rüdiger, S., Germeroth, L., Schneider-Mergener, J., and Bukau, B. (1997). Substrate specificity of the DnaK chaperone determined by screening cellulosebound peptide libraries. EMBO J. 16, 1501-1507. doi: 10.1093/emboj/16. 7.1501

Sanz, E., and Marenduzzo, D. (2010). Dynamic Monte-Carlo versus Brownian dynamics: a comparison for self-diffusion and crystallization in colloidal fluids. J. Chem. Phys. 132, 194102. doi: 10.1063/1.3414827

Schmid, D., Baici, A., Gehring, H., and Christen, P. (1994). Kinetics of molecular chaperone action. Science 263, 971-973. doi: 10.1126/science. 8310296

Scidmore, M. A., Okamura, H. H., and Rose, M. D. (1993). Genetic interactions between KAR2 and SEC63, encoding eukaryotic homologues of DnaK and DnaJ in the endoplasmic reticulum. Mol. Biol. Cell 3, 1145-1159. doi: $10.1091 / \mathrm{mbc} .4 .11 .1145$

Shalgi, R., Hurt, A. J., Krykbaeva, I., Taipale, M., Lindquist, S., and Burge, C. B. (2013). Widespread regulation of translation by elongation pausing in heat shock. Mol. Cell 49, 439-452. doi: 10.1016/j.molcel.2012. 11.028

Soranno, A., Buchli, B., Nettels, D., Cheng, R. R., Müller-Späth, S., Pfeil, S. H., et al (2012). Quantifying internal friction in unfolded and intrinsically disordered proteins with single-molecule spectroscopy. Proc. Natl. Acad. Sci. U.S.A. 109, 17800-17806. doi: 10.1073/pnas.1117368109

Tiana, G., Sutto, L., and Broglia, R. A. (2007). Use of the metropolis algorithm to simulate the dynamics of protein chains. Physica A 380, 241-249. doi: 10.1016/j.physa.2007.02.044 
van Kampen, N. G. (1992). Stochastic Processes in Physics and Chemistry. NorthHolland; Amsterdam: Elsevier.

Wilcox, A. J., Choy, J., Bustamante, C., and Matouschek, A. (2005). Effect of protein structure on mitochondrial import. Proc. Natl. Acad. Sci. U.S.A. 102, 15435-15440. doi: 10.1073/pnas.0507324102

Zhu, X., Zhao, X., Burkholder, W. F., Gragerov, A., Ogata, C. M., Gottesman, M. E., et al. (1996). Structural analysis of substrate binding by the molecular chaperone DnaK. Science 272, 1606-1614. doi: 10.1126/science.272.5268.1606

Zuiderweg, E. R. P., Bertelsen, E. B., Rousaki, A., Mayer, M. P., Gestwicki, J. E., and Ahmad, A. (2013). Allostery in the Hsp70 chaperone proteins. Top. Curr. Chem. 328, 99-153. doi: 10.1007/128_2012_323
Conflict of Interest Statement: The authors declare that the research was conducted in the absence of any commercial or financial relationships that could be construed as a potential conflict of interest.

Copyright (c) 2015 Assenza, De Los Rios and Barducci. This is an open-access article distributed under the terms of the Creative Commons Attribution License (CC $B Y)$. The use, distribution and reproduction in other forums is permitted, provided the original author(s) or licensor are credited and that the original publication in this journal is cited, in accordance with accepted academic practice. No use, distribution or reproduction is permitted which does not comply with these terms. 